\title{
Variations
}

Variations

Revue internationale de théorie critique

$13 / 14 \mid 2010$

Le choix du petit

\section{La lutte des classes chez Marx : reconnaissance ou dénégation?}

L'analyse critique de Castoriadis

\section{Nicolas Poirier}

\section{(2) OpenEdition}

\section{Journals}

Édition électronique

URL : http://journals.openedition.org/variations/180

DOI : $10.4000 /$ variations. 180

ISSN : 1968-3960

Éditeur

Les amis de Variations

Édition imprimée

Date de publication : 31 mars 2010

Référence électronique

Nicolas Poirier, "La lutte des classes chez Marx : reconnaissance ou dénégation? 》, Variations [En ligne], 13/14 | 2010, mis en ligne le 01 février 2012, consulté le 30 avril 2019. URL : http:// journals.openedition.org/variations/180; DOI : 10.4000/variations. 180

Ce document a été généré automatiquement le 30 avril 2019

Les ami•e•s de Variations 


\title{
La lutte des classes chez Marx: reconnaissance ou dénégation?
}

L'analyse critique de Castoriadis

\author{
Nicolas Poirier
}

\section{NOTE DE L'ÉDITEUR}

Première publication sur www.theoriecritique.com, « Le choix du petit », Printemps 2010, pp. 74-90

1 L'originalité de la lecture de Marx par Castoriadis, développée principalement dans le cadre de la revue Socialisme ou Barbarie, et il faut aussi le reconnaître son caractère problématique, est de montrer, à rebours de la vulgate marxiste, que la critique de l'économie capitaliste élaborée par Marx dans Le Capital n'a été en fait rendue possible que moyennant l'élimination d'un facteur auquel Marx attribue pourtant un rôle fondamental: la lutte des classes. Du coup, si Marx doit renoncer à intégrer dans son raisonnement le facteur de la lutte des classes autrement que comme une simple variable d'ajustement ne changeant rien aux tendances lourdes de l'économie capitaliste, c'est parce que, d'après Castoriadis, cela contredirait les présupposés déterministes au fondement de sa réflexion et l'obligerait à prendre en compte la dimension proprement historique de l'évolution économique et sociale, mettant ainsi en lumière le potentiel créateur d'actions humaines susceptibles de faire surgir ce qui n'était pas inscrit dans la stricte fonctionnalité du système économique ${ }^{1}$ : "C'est en effet comme des purs et simples objets qu'ouvriers et capitalistes apparaissent dans Le Capital. Ils n'y sont que les instruments aveugles et inconscients réalisant par leurs actes ce que les "lois économiques" imposent. Si l'économie doit devenir une mécanique de la société il faut qu'elle ait affaire à des phénomènes régis par des lois “objectives”, indépendantes de l'action des hommes et des classes. On aboutit ainsi à un énorme paradoxe : Marx, qui a découvert la lutte des classes, écrit un ouvrage monumental analysant le développement 
du capitalisme, ouvrage d'où la lutte des classes est totalement absente ${ }^{2}$.» Ce que Castoriadis critique dans l'analyse de Marx, ce n'est pas tel ou tel point qu'il aurait convenu de corriger, mais le principe même d'une théorie qui, s'interdisant de prendre en considération la lutte des classes autrement que comme un facteur marginal, ne peut en définitive penser une action autonome du prolétariat susceptible d'échapper aux implacables lois mécaniques gouvernant l'économie capitaliste ${ }^{3}$.

2 Une lecture attentive de la pensée de Marx conduit à relever dans celle-ci un certain nombre d'ambiguïtés qui auraient, d'après Castoriadis, conduit le marxisme à osciller sans cesse entre une vision déterministe de l'histoire selon laquelle le capitalisme serait nécessairement conduit à s'effondrer en vertu de ses lois intrinsèques, et une conception de la praxis historique qui accorde à l'action des hommes en tant que telle un rôle moteur dans le devenir des sociétés. Or Castoriadis ne se contente pas de constater ces équivoques, qui sont après tout le propre des grandes pensées, mais tranche dans le sens d'un Marx déterministe et objectiviste, la dimension « praxiste » et révolutionnaire de la pensée de Marx ayant été recouverte chez Marx lui-même par la dimension «théoriciste et spéculative, telle qu'elle s'exprimerait de manière emblématique dans l'avant-propos à la Critique de l'économie politique. La pensée de Marx est en effet l'objet d'une critique qui se développe chez Castoriadis selon une double perspective : d'abord, sur un plan que l'on pourrait qualifier de pratique, Marx ne verrait dans la contradiction interne au capitalisme que celle impliquée par le développement des forces productives tendant à faire éclater l'enveloppe trop étroite des rapports de production capitalistes, ce qui le rendrait aveugle à l'antagonisme fondamental du mode de production capitaliste qui sépare les processus de direction et les mécanismes d'exécution, sollicitant l'autonomie des travailleurs qu'il tend dans le même mouvement à détruire ${ }^{4}$. Ensuite, sur un plan plus théorique, Marx resterait prisonnier de l'antinomie de la liberté et de la nécessité, qui le conduirait à concevoir à la fois l'action du prolétariat comme intégralement déterminée par les lois de l'histoire et comme déterminant librement ces mêmes lois - soumis à la plus extrême des réifications, le prolétariat s'affirmerait comme le sujet libre et conscient de sa propre histoire. Or selon Castoriadis la signification profonde de la praxis révolutionnaire ne consiste pas pour le prolétariat à n'être que l'instrument aveugle de la raison historique, mais à faire son histoire dans des conditions déterminées sur lesquelles il peut agir tout en se transformant lui-même ${ }^{5}$.

3 La critique de Castoriadis ne se borne donc pas à l'examen de la théorie de la valeur chez Marx, afin de répondre à la question de savoir si la force de travail est bel et bien une marchandise comme une autre dont la valeur peut être déterminée objectivement, ou si elle constitue au contraire un élément du système capitaliste rigoureusement indéterminé, renvoyant à une capacité de résistance des travailleurs qui atteste que le « destin » de l'économie libérale n'est pas scellé mais qu'il repose sur un conflit - la lutte des classes - dont on ne peut entrevoir d'issue a priori dans un sens (exploitation accrue des travailleurs) ou dans un autre (baisse du taux d'exploitation débouchant à terme sur une révolution socialiste). Elle porte plus largement sur le fondement même de l'économie capitaliste, et sur le sens et le contenu que l'on doit donner au projet révolutionnaire : de ce point de vue, une révolution socialiste ne saurait se limiter d'après Castoriadis à abolir le principe de l'exploitation économique, elle doit avant tout redonner aux salariés la direction de leur travail et plus largement de l'économie, en abolissant la distinction entre dirigeants et exécutants, dans la mesure où une telle dichotomie est la matrice des rapports de domination capitaliste. Nous chercherons donc 
ici à examiner les équivoques de la pensée de Marx concernant la détermination de la valeur de la force de travail et plus largement le problème de la lutte des classes, avant de mesurer l'apport de Castoriadis à la critique de l'organisation capitaliste du travail.

\section{La détermination de la valeur de la force de travail}

4 Selon Castoriadis, Marx cherche à montrer qu'il existe une valeur objective de la force de travail, et qu'en réussissant à déterminer celle-ci dans le cadre des lois de l'économie de marché concurrentielle, il est possible de faire ressortir les tendances structurelles du capitalisme : l'augmentation du taux d'exploitation, conjuguée à la baisse inéluctable à terme du taux de profit, révéleraient de façon immanente les contradictions objectives qui minent le capitalisme et doivent le conduire nécessairement à son effondrement ${ }^{6}$. L'accroissement du capital productif qui se traduit, d'après Marx, par une accélération du processus de division sociale du travail, doublé d'un développement du machinisme à grande échelle, a en effet pour conséquence l'intensification de la concurrence entre les travailleurs et la baisse continuelle de leur salaire: le masse du prolétariat tendant à croître de façon exponentielle, l'augmentation du rythme de fréquence des crises, ainsi que de leur violence, devient la seule issue d'un processus conduisant à l'effondrement du système. Ainsi, afin de prouver que dans un système d'économie capitaliste l'exploitation des travailleurs ne peut que croître ${ }^{7}$, suivant le postulat selon lequel « profit et salaires sont en raison inverse l'un de l'autre ${ }^{8}$ " - qu'autrement dit le taux d'exploitation ${ }^{9}$ (rapport de la masse des profits à la masse des salaires) est en augmentation constante et se traduit par une misère croissante du prolétariat ${ }^{10}$, Marx va chercher à montrer que la valeur de la force de travail est objectivement déterminable au même titre que n'importe quelle marchandise ${ }^{11}$ : tout se passe comme si le capitaliste pouvait être sûr d'extraire de l'ouvrier le maximum de rendement possible, de la même manière qu'il sait a priori ce qu'il peut extraire comme quantité de calories d'une tonne de charbon.

Or, l'expérience de la lutte des classes montre d'après Castoriadis que la valeur d'usage de la force de travail (ce que le capitaliste peut tirer du travail ouvrier) n'est pas déterminable indépendamment du rapport de force existant entre le patron et l'ouvrier, et que la détermination du taux de rendement fait l'objet d'une confrontation quotidienne dans le cadre de l'usine: le taux de rendement sera d'autant moins élevé que la résistance délibérée opposée par les travailleurs aux normes de production sera plus intense : « La production, écrit Castoriadis, loin d'être intégralement déterminée par la volonté du capitaliste individuel d'augmenter le rendement du travail, est tout autant déterminée par la résistance individuelle et collective des ouvriers à cette augmentation. L'extraction de la valeur d'usage de la force de travail n'est pas une opération technique, mais un processus de lutte acharnée, dans lequel les capitalistes se retrouvent perdants pour ainsi dire une fois sur deux ${ }^{12}$.»

6 Le même raisonnement tient pour la valeur d'échange de la force de travail : là encore, il n'y a pas de valeur d'échange de la force de travail qui puisse être définie indépendamment de la lutte quotidienne des ouvriers contre l'exploitation. Le salaire versé à l'ouvrier afin de lui permettre d'assurer la reproduction quotidienne de sa force de travail est fonction du rapport de force travailleurs/capitalistes; en fait, ce qu'un entrepreneur achète, ce n'est pas l'heure de travail d'un ouvrier mais le rendement effectif que pourra fournir cet ouvrier durant un laps de temps d'une heure, - autrement dit un potentiel de productivité (la valeur d'usage de la force de travail) qui est, on vient 
de le voir, indéterminé: c'est pourquoi là aussi, la détermination du salaire fait l'objet d'une lutte acharnée entre patrons et ouvriers, qui ne peut être détachée de la lutte pour la détermination du taux de rendement ${ }^{13}$. La lutte des classes constitue donc le présupposé de tout rapport d'exploitation, puisque c'est à partir du conflit primordial pour l'établissement de la durée de la journée de travail qu'est déterminé le temps de travail nécessaire à la reproduction de la force de travail ${ }^{14}$ : loin d'être la résultante des lois objectives de l'économie capitaliste, qui verrait le taux d'exploitation du travail ouvrier augmenter parallèlement à la hausse des gains de productivité, la valeur objective de la force de travail est en réalité déterminée de façon essentielle par le niveau du rapport de force existant à un moment donné entre le capital et le travail qui ne peut être formalisé, sinon que sur un mode tendanciel, par un système de lois fixes et sur la base d'invariances structurelles. La lutte des classes rentre ainsi pour une large part dans la détermination de la valeur d'échange de la force de travail (« tant d'argent pour une heure de travail ») et de sa valeur d'usage («tant de productivité pour une heure de travail ») ${ }^{15}$. Comme peut l'écrire Castoriadis: "La force de travail ne peut jamais devenir marchandise pure (malgré les efforts du capitalisme). Il n'y a pas de valeur d'échange de la force de travail déterminée par des facteurs "objectifs" le niveau des salaires est essentiellement déterminée par les luttes ouvrières formelles et informelles. Il n'y a pas de valeur d'usage définie de la force de travail, la productivité est l'enjeu d'une lutte incessante dans la production, dont l'ouvrier est un sujet actif autant que passif ${ }^{16}$.»

\section{La lutte des classes chez Marx : une reconnaissance...}

7 Les positions de Castoriadis semblent assez déconcertantes puisqu'on peut lire à de nombreuses reprises sous la plume de Marx des passages où celui-ci montre au contraire que la lutte des classes joue un rôle important dans la détermination du taux d'exploitation du travail salarié ${ }^{17}$, la résistance des travailleurs influant très largement sur les conditions de répartition entre temps de travail nécessaire et surtravail, par conséquent sur la détermination du profit, c'est-à-dire cette partie impayée du travail ouvrier. S'il est tout à fait certain que Marx a bel et bien reconnu le principe de cette lutte entre le capital et le travail, les travailleurs se battant pour une hausse des salaires et une réduction du temps de travail contre la tendance inverse du capital à accentuer le taux de profit en baissant les salaires, il est cependant loin d'être évident, et c'est là le sens des objections de Castoriadis sur ce point, qu'il lui ait accordé une place centrale dans la dynamique du capitalisme, émettant plutôt de sérieux doutes sur sa capacité à contrecarrer le mouvement du capital à l'appropriation d'une partie toujours plus grande du produit social. La conclusion du texte de 1865 Salaire, prix et plus-value ${ }^{18}$ est à cet égard tout à fait révélatrice et rentre ainsi sous le coup de la critique faite par Castoriadis.

8 À la question de savoir jusqu'à quel point le travail peut opposer une résistance durable au capital qui l'oblige à aller dans le sens des exigences du mouvement ouvrier ${ }^{19}$, Marx commence par faire observer que la valeur de la force du travail a ceci de bien particulier, qu'à la différence des autres marchandises dont la valeur reste relativement constante, elle n'est pas une grandeur fixe mais est au contraire sujette à des variabilités, et que c'est la lutte entre le capital et le travail pour la détermination du taux de profit qui explique en partie cette instabilité structurelle de la valeur du travail ${ }^{20}$. En effet, sans même se référer aux différences culturelles impliquant que le prix du travail se trouve déterminé dans des conditions nationales chaque fois particulières par un standard de vie qui est 
tout sauf universel ${ }^{21}$, les simples conditions physiques dont le respect permet la reproduction journalière du travail ne valent elles-mêmes que de façon relativement flottante, et c'est pourquoi leur contenu effectif résulte aussi du degré de résistance physique des ouvriers à l'exploitation qui est tout à fait variable. Bien entendu, il est impossible de faire travailler un homme au-delà d'un certain seuil qui ne peut être reculé à l'infini ${ }^{22}$, en ce sens il y a des limites physiques à l'exploitation économique, mais il faut néanmoins constater que la journée de travail par elle-même peut fluctuer entre des durées maximales et minimales qui ne sont pas définies en vertu des seules possibilités et impossibilités physiques ${ }^{23}$. Sans aller jusqu'à suivre l'hypothèse de Marx selon laquelle le marché du travail pourrait tout aussi bien fonctionner avec des esclaves exploités à leurs plus extrêmes limites qu'avec des travailleurs correctement rémunérés ${ }^{24}$, un simple aperçu historique oblige à voir dans les formes très diverses prises historiquement par l'exploitation économique avant tout les résultantes conjoncturelles du conflit de classe, qui vient se cristalliser en des formations de compromis toujours instables et fonctionnant sur la base d'un équilibre précaire. C'est dans une telle perspective que s'inscrit précisément la reconnaissance par Marx de la lutte des classes comme une condition déterminante de l'évolution de l'économie capitaliste : «Nous pouvons dire [...] que les limites de la journée de travail étant données, le maximum du profit correspond au minimum physiologique du salaire; et que le salaire étant donné, le maximum du profit correspond à un allongement de la journée de travail qui soit compatible avec les forces physiques de l'ouvrier. Il est évident qu'entre les deux limites de ce taux maximal du profit, une vaste échelle de variations est concevable. La fixation de son degré réel ne peut s'établir que dans la lutte continuelle du capital et du travail: le capitaliste tend constamment à réduire le salaire à son minimum physiologique, et à prolonger la journée de son travail jusqu'au maximum physiologique ; le travailleur fait constamment pression dans le sens opposé25. » Quelle que soit donc la tendance structurelle du capitalisme à abaisser le niveau de vie de la classe ouvrière à sa limite la plus basse, les travailleurs ne doivent en aucun cas selon Marx renoncer aux efforts pour résister à cette entreprise d'expropriation, car inséparables du système salarial, les luttes pour la réduction de la journée du travail et la hausse des salaires sont nécessaires au maintien de la valeur du travail à un niveau d'équilibre moyen ${ }^{26}$.

\section{... ou une dénégation?}

9 Mais si la prise en compte de la lutte des classes vaut d'une certaine manière chez Marx comme une reconnaissance de l'historicité du capitalisme, reste que cet apport nécessaire à la compréhension du fait économique et social est resté d'après Castoriadis contradictoire, et se serait heurté à une autre dimension de la pensée de Marx, qui ne concevrait plus la perspective révolutionnaire selon une logique du conflit irréductible aux explications de type mécaniste, mais dans une optique intégralement déterministe où le système de l'économie politique serait pensé sur le modèle de la science physique ${ }^{27}$. Il est clair qu'à ce jeu les catégories de l'économie qui doivent constituer, pour garder leur potentiel critique, de simples indicateurs opératoires sans prétention à la substantialité, se transforment immanquablement en des concepts réifiés qui cherchent à traduire les lois immanentes de l'expansion capitaliste, au mépris de la distinction incontournable du possible et du réel, en montrant que quoi que l'on puisse penser ou faire, la logique de l'économie capitaliste ne peut conduire crises après crises qu'à son effondrement. Dès 
lors donc qu'on établit des relations fonctionnelles rigoureusement déterminées entre des entités posées comme invariantes, l'idée même d'une lutte ou d'une simple résistance autre que physique perd en effet toute signification, puisque ce ne sont plus des acteurs conscients de leurs faits et gestes qui viennent à se confronter, mais des choses régies par des lois mécaniques dont elles sont le jouet: «... Cette conception équivaut, écrit Castoriadis, à traiter dans la théorie les ouvriers comme le capitalisme voudrait mais ne peut pas les traiter dans la pratique de la production - à savoir comme des objets purs et simples. Elle équivaut à dire que la force de travail est intégralement marchandise, au même titre qu'un animal, un combustible ou un minerai. Elle possède une valeur d'échange qui correspond à son coût objectif déterminé par les forces du marché ; elle possède une valeur d'usage dont l'extraction ne dépend que du bon vouloir du capitaliste et de ses méthodes de production. Le charbon ne peut pas influer sur le prix auquel il est vendu; ni empêcher le capitaliste d'augmenter son rendement énergétique par des méthodes d'utilisation perfectionnées. L'ouvrier non plus $^{28}$. » Dans le cadre d'une division accrue du travail qui accroît la concurrence entre les salariés et d'un développement technique qui tend à remplacer le travail vivant par des machines, la valeur de la force de travail ne pourrait finalement que diminuer à mesure que s'élève la productivité du travail ${ }^{29}$, ce qui est logique puisque si la valeur de la force de travail est déterminée d'après la quantité de travail nécessaire pour la reproduire, la rémunération de la quantité de travail sera d'autant moindre que le temps de travail incorporé dans chaque marchandise diminue en raison du progrès technique. C'est pourquoi Marx peut affirmer que les travailleurs ne doivent pas se faire d'illusions sur la possibilité de résister au mouvement d'accumulation capitaliste par lequel le travail mort se nourrit de la force de travail vivante et l'aliène à sa logique mortifère : la lutte contre l'exploitation ne peut que ralentir un tel processus, en aucun cas en renverser le cours - si les prolétaires peuvent dans une certaine mesure "résister", ce sont seulement contre les effets, jamais contre les causes de ces effets qui s'enchaînent inexorablement d'après des connexions quasiinvariables ${ }^{30}$. Comme l'écrira Marx au livre III du Capital, «la production en vue de la valeur et de la plus-value implique [...] la tendance, toujours opérante, à réduire audessous de la moyenne sociale existante le temps de travail nécessaire à la production d'une marchandise, c'est-à-dire sa valeur ${ }^{31}$. »

D'où la critique de Marx par Castoriadis : obnubilé par la catégorie de "marchandise », Marx aurait fait de la tendance constante du capitalisme à la réification des ouvriers l'état habituel et constitutif de l'économie capitaliste, sans voir que si la réduction des travailleurs au rang de simples choses devenait effective, l'économie s'effondrerait aussitôt, puisque la possibilité de créer une plus-value, source d'investissement et de profits futurs, serait détruite ${ }^{32}$. C'est là d'ailleurs la contradiction d'un tel système soumis à l'obligation de solliciter la participation des exécutants au processus de production dans l'objectif de sa rationalisation, tout en leur interdisant de prendre la moindre initiative qui irait dans le sens d'une transformation du rapport du travailleur à son objet sous l'angle de la créativitée ${ }^{33}$ : il est à la fois nécessaire à la survie du système que les travailleurs gèrent eux-mêmes leurs activités à rebours des ordres donnés par la direction, puisque c'est justement la condition pour que l'appareil de production atteigne un degré de fonctionnalité qui le rende viable à terme, et qu'ils ne puissent pas en même temps prendre part de manière active au processus de production, car cela ouvrirait sinon une brèche dans l'organisation bureaucratique de l'entreprise et signifierait que 
c'est aux producteurs eux-mêmes que doit revenir la responsabilité légitime de la direction du travail ${ }^{34}$.

\section{Le caractère spécifique de la domination capitaliste selon Castoriadis}

11 On ne doit donc pas, d'après Castoriadis, se méprendre sur l'intention de Marx : lorsque celui-ci entend montrer que la dynamique objective des contradictions du capitalisme résulte du conflit entre le développement des forces productives et la baisse du niveau de consommation ouvrière empêchant les prolétaires de jouir du fruit de leur travail ${ }^{35}$, il fait seulement ressortir une dimension effectivement centrale de l'économie capitaliste qu'il traduit en termes de lois objectives, mais s'interdit de mettre le doigt sur la contradiction majeure du capitalisme moderne, qui ne vient pas uniquement de la lutte pour la détermination de la valeur du travail puisqu'elle concerne la totalité de l'organisation de la production elle-même, et ne peut être simplement pensée dans la perspective d'une tendance inexorable à la paupérisation générale. Si le travail est exploité, c'est avant tout pour Castoriadis dans l'exacte mesure où l'ouvrier est exproprié de la direction de ses activités, l'extorsion de la plus-value n'étant qu'un mode de cette aliénation première ${ }^{36}$.

Castoriadis montre ainsi que le véritable antagonisme ne concerne pas tant la répartition du produit social que l'organisation des rapports de production au sein de l'entreprise elle-même : de ce point de vue, le capitalisme serait la première société de l'histoire à être bâtie sur une contradiction interne insurmontable, à la différence des sociétés esclavagistes ou féodales, certes marquées par des oppositions indéniables, mais dont l'organisation n'est pas comme telle contradictoire ${ }^{37}$; en effet, quelle que soit la violence du conflit opposant le maître à son esclave, dont le niveau d'intensité peut parfois être considérable, une société fondée sur la domination de caste forme une totalité cohérente où chacun des membres, en remplissant la fonction qui lui est propre, concourt à la perpétuation de l'ensemble - si une éventuelle contestation par les dominés du caractère illégitime du pouvoir des maîtres est toujours possible, celle-ci ne dépasse pas le stade de la révolte ponctuelle et peut donc être comprimée dans les limites du système de domination ${ }^{38}$. Quelle que soit l'asymétrie profonde des différentes positions dans l'espace social, il n'en reste donc pas moins que dans de telles sociétés, le système global de légitimation du pouvoir fournit à l'ensemble des groupes et individus des repères identificatrices sans commune mesure avec la place qu'ils occupent dans le système de distribution du produit social - dans ces conditions il est logique que le phénomène de l'exploitation soit massivement occulté, puisque les dominants qui exploitent la force de travail des « classes inférieures » justifient leurs privilèges pour des raisons qui sont tout sauf économiques.

Un texte inédit de Castoriadis du début des années 1960 exprime bien cette idée: "L'asymétrie du schème imaginaire est fréquemment recouverte par son "intégration" dans un schème cosmique ou théologique formellement plus général, où paria et brahman sont également soumis au Dharma, pauvres et riches renvoyés à l'amour égal de Dieu pour ses créatures, ou bien elle est travestie par des rationalisations qui font appel à la fonction "organique" de cette asymétrie et à la complémentarité des parties dissemblables de la société : la fable de Menenius Agrippa en est le premier exemple enregistré dans l'histoire occidentale. "Vous fournissez le travail, nous fournissons 
l'honneur, la sainteté, les idées", "Vous êtes les bras, nous sommes le cerveau", est le type général de ces rationalisations ${ }^{39}$. » On dira donc que dans de telles formations sociales, les oppositions étant régulées sur un plan symbolique, il ne peut exister de dialectique commune au travers de laquelle les antagonismes deviendraient des contradictions véritables, c'est-à-dire des schèmes de confrontation dans lesquels l'action de chacun des adversaires serait dirigée contre l'autre et l'obligerait à changer son mode de comportement : «Ce passage à une structure asymétrique et objectivement antagonique, écrit Castoriadis dans le même texte, à une division en classes, équivaut à une modification profonde aussi bien des moments imaginaires que des moments réels de la situation sociale. Le schème imaginaire du "nous" articulé qui sous-tend la vie d'une société archaïque est symétrique, c'est-à-dire fonde une réciprocité. Le passage à une division en classes introduit l'asymétrie dans le schème imaginaire et fait disparaître la réciprocité dans les situations réelles ${ }^{40}$. »

La société capitaliste serait donc construite, d'après les analyses de Castoriadis, sur une contradiction intrinsèque, semblable à celle qui structure le psychisme d'un névrosé : l'organisation capitaliste du travail n'est fonctionnelle que dans la mesure même où les opérations nécessaires à sa perpétuation vont à l'inverse de son intention initiale ${ }^{41}$. La direction bureaucratique doit conformément à son objectif de rationalisation intégrale du travail, qui passe par la quantification de l'activité productrice et donc par son démembrement en une série d'opérations mécaniques, réduire les salariés au rang de simples exécutants ${ }^{42}$, mais doit dans le même mouvement s'appuyer sur le potentiel créateur du travail ouvrier, et donc nier sa qualité de direction séparée cherchant à imposer de l'extérieur l'organisation du travail : à partir du moment où elle fait appel à une qualification strictement humaine, l'exécution d'une directive élaborée a priori mobilise nécessairement ce que Castoriadis appelle un "élément d'auto-direction ${ }^{43}$ ", et ne peut en tant que telle être assimilée à une exécution pure et simple, à la manière d'une machine qui opérerait à partir de sa programmation initiale. Dans ces conditions, le capitalisme ne peut fonctionner que parce que les travailleurs opposent une résistance à son mode d'organisation: ce n'est que dans la mesure où les salariés organisent euxmêmes leur travail et opposent à la planification bureaucratique de leurs tâches une contre-organisation informelle par laquelle ils peuvent contrôler leur activité que la production est justement la plus efficace possible ${ }^{44}$. La direction bureaucratique de la production est donc contradictoire en ce sens qu'elle doit s'appuyer sur ce que l'organisation capitaliste du travail tend précisément à supprimer - la faculté d'autoorganisation des collectivités humaines et la créativité des individus: «La direction capitaliste [...] prétend réduire l'ouvrier à des tâches limitées et déterminées, mais elle est en même temps obligée de s'appuyer sur les capacités universelles que celui-ci développe à la fois en fonction et à l'encontre de la situation qui lui est faite. De ces tâches, elle prétend enlever tout élément de direction en définissant d'avance exhaustivement les modalités d'exécution; mais cette définition exhaustive étant toujours impossible, la production ne peut être effectuée que dans la mesure où l'ouvrier organise lui-même son travail et dépasse le rôle du pur et simple exécutant qui est théoriquement le $\operatorname{sien}^{45}$. » 


\section{L'auto-organisation de la production : un projet révolutionnaire}

15 Les positions de Castoriadis ne sont ici pas très éloignées de ce que la tradition du communisme des conseils désignera sous les termes d'« auto-organisation » de la classe ouvrière ou de «créativité » prolétarienne, même si Castoriadis se montrera très critique envers le refus manifeste du conseillisme à prendre en compte la nécessité de l'institution politique. Castoriadis insiste en effet très nettement sur l'idée suivant laquelle le projet socialiste est inconcevable s'il ne repose pas sur la praxis effective des travailleurs, dont l'action consistant à organiser le travail dans l'entreprise bureaucratique à rebours des directives officielles, contient les prémisses d'une gestion démocratique de la production et de la société. Selon Castoriadis, si Marx a bien fait ressortir le caractère aliénant du travail dans le cadre du capitalisme, en mettant en évidence le fait que l'objectif du capital est de faire fructifier le travail vivant par le travail mort, son analyse des formes concrètes prises par l'aliénation est restée partielle ${ }^{46}$, faute d'avoir pu saisir le caractère contradictoire de l'organisation capitaliste de la production qui tend à réifier la force de travail tout en utilisant les capacités créatrices des travailleurs sans lesquelles le système s'effondrerait, et certainement pas pour des raisons de baisse tendancielle du taux de profit ou d'élévation de la composition organique du capital.

L'effort de Marx pour transformer la valeur d'usage et la valeur d'échange de la force de travail en des variables déterminées par les lois objectives de l'économie aboutirait selon Castoriadis à des contradictions insurmontables, et il faudrait alors en conclure à la pérennité du capitalisme : si en effet la force de travail était intégralement réifiée, comme le laisse supposer Marx, le capitalisme ne rencontrerait du coup nulle résistance de la part des travailleurs et ne connaîtrait aucune des crises dont Marx dit pourtant qu'elles sont les facteurs conditionnant l'effondrement à terme du système ${ }^{47}$.

Or loin d'être réductible à une simple variable d'ajustement ne modifiant qu'à la marge, et encore, l'équilibre général du système d'exploitation de la force de travail, la lutte des ouvriers contre l'expropriation de leur travail constitue le principe déterminant duquel il faut partir si l'on veut comprendre dans quelle mesure une transformation socialiste des rapports de production est non seulement possible mais aussi et surtout réalisable : si le passage du capitalisme au communisme via le socialisme n'est pas un projet utopique mais une réalité inscrite dans le mouvement de contestation du système de domination existant, c'est qu'en résistant à l'exploitation de leur force de travail, les ouvriers posent les bases d'une auto-organisation rationnelle de la production qui constitue en quelque sorte l'aspect positif de leur lutte contre la réification :

18 «L'oppression, l'exploitation, l'aliénation créées par le capitalisme s'expriment chez la classe ouvrière par des contradictions que jusqu'ici elle n'est pas arrivée à surmonter. La positivité de la classe ouvrière c'est qu'elle ne reste pas simplement déchirée par ces contradictions, mais qu'elle lutte constamment pour les dépasser et, que aux niveaux les plus différents, le contenu de cette lutte est l'organisation autonome des ouvriers, la gestion ouvrière de la production, finalement la réorganisation de la société [...] La lutte du prolétariat n'est pas et ne peut pas être simplement une lutte "contre" l'exploitation ; elle tend nécessairement à être une lutte pour une nouvelle organisation des rapports de production $^{48}$. " 


\section{NOTES}

1. Voir « Prolétariat et organisation, I », dans L'Expérience du mouvement ouvrier, T. 2, Paris, UGE, «10-18», 1974, p. 146.

2. "Le mouvement révolutionnaire sous le capitalisme moderne", dans Capitalisme moderne et révolution, T. 2, Paris, UGE, « 10-18», 1979, p. 102.

3. Voir ibid.

4. Voir «Recommencer la révolution", dans L'Expérience du mouvement ouvrier, T. 2, op. cit., p. 318.

5. Voir ibid., p. 318-319.

6. Voir notamment Travail salarié et capital, Euvres. Economie I, Paris, Gallimard, Pléiade, p. 216-229.

7. Voir « Le mouvement révolutionnaire sous le capitalisme moderne », op. cit., p. 77.

8. Travail salarié et capital, op. cit., p. 221.

9. Voir Le Capital, Livre premier, deuxième section, chapitre IX, Euvres. Economie I, op. cit., p. 770-772.

10. Voir Travail salarié et capital, op. cit., p. 219.

11. Voir ibid., p. 206.

12. "Le mouvement révolutionnaire sous le capitalisme moderne", op. cit., p. 85-86. Voir également "Pourquoi je ne suis plus marxiste », dans Une société à la dérive, Paris, Seuil, 2005, p. 46-47.

13. Voir ibid., p. 47.

14. C'est d'ailleurs ce que reconnaît Marx lui-même dans Le Capital (voir Livre premier, troisième section, chapitre X, II, op. cit., p. 791).

15. Voir «Le mouvement révolutionnaire sous le capitalisme moderne», op. cit., p. 86. Voir également "L'histoire du mouvement ouvrier", dans L'Expérience du mouvement ouvrier, T. 1, Paris, UGE, « 10-18 », 1974, p. 89-90.

16. «Recommencer la révolution », op. cit., p. 318.

17. Daniel Bensaïd affirme ainsi que la lutte des classes constitue chez Marx le fil conducteur de sa compréhension de l'économie politique, contrairement aux allégations de Castoriadis qui soulignerait au contraire l'aveuglement supposé de Marx quant à l'effet des luttes ouvrières sur la répartition du produit social : voir «Politiques de Castoriadis", dans Cornelius Castoriadis. Réinventer l'autonomie, collectif, Paris, Sandre, 2008, p. 258.

18. Euvres. Economie I, op. cit., p. 473-533.

19. Voir ibid., p. 527.

20. Voir ibid., p. 529.

21. Voir ibid., p. 528 ; Le Capital, Livre premier, troisième section, chapitre X, II, op. cit., p. 788.

22. Voir ibid., p. 787-788; voir aussi Salaire, prix et plus-value, op. cit., p. 528.

23. Voir ibid.; voir également Le Capital, ibid., p. 788.

24. Voir Salaire, prix et plus-value, op. cit., p. 528.

25. Ibid., p. 529.

26. Voir ibid., p. 532 : «Je crois avoir montré que les luttes pour des salaires normaux sont des incidents inséparables du système des salaires dans son ensemble, que dans 99 cas sur cent, les travailleurs, en s'efforçant de relever les salaires, s'évertuent tout simplement pour soutenir la valeur donnée du travail, que la nécessité de débattre de leur prix avec le capitaliste est inhérente à leur condition, qui les contraint de se vendre comme des marchandises. Lâcher prise 
sans courage, dans ce conflit de chaque jour avec le capital, ce serait perdre la faculté de se lancer un jour dans un mouvement plus vaste. " (Je souligne)

27. Voir « Prolétariat et organisation, I », op. cit., p. 145-146 ; voir également « Pourquoi je ne suis plus marxiste ", op. cit., p. 53

28. «Le mouvement révolutionnaire sous le capitalisme moderne », op. cit., p. 85.

29. Voir Salaire, prix et plus-value, op. cit., p. 532.

30. Voir ibid.: «... Tout à fait en dehors de la servitude générale qu'implique le système des salaires, les travailleurs ne doivent pas s'exagérer le résultat final de ces luttes quotidiennes. Qu'ils ne l'oublient pas : ils combattent les effets, non pas les causes ; ils retardent la descente, ils n'en changent point la direction; ils appliquent des palliatifs, mais ne guérissent pas la maladie. Qu'ils aient garde de se laisser prendre tout entiers à ces escarmouches inévitables que provoquent chaque nouvel empiétement du capital, chaque variation du marché. »

31. Le Capital, livre III, septième section, chapitre XXVIII, p. 1479.

32. Voir "Sur le contenu du socialisme, II », dans Le Contenu du socialisme, Paris, UGE, «10-18 », 1979, p. 132 : «Dans Le Capital - par opposition à ses écrits de jeunesse - il n'apparaît guère que le prolétariat est - et ne peut qu'être - porteur positif de la production capitaliste qui est obligée de s'appuyer sur lui comme tel et de le développer comme tel en même temps qu'elle essaie de le réduire à un rôle purement mécanique et à la limite de l'expulser de la production. De ce fait même, cette analyse ne voit pas que la crise première du capitalisme est cette crise dans la production, découlant de l'existence simultanée de deux tendances contradictoires dont aucune ne saurait disparaître sans que le capitalisme s'effondre. »

33. Voir « Le mouvement révolutionnaire sous le capitalisme moderne », op. cit., p. 147.

34. Voir «Sur le contenu du socialisme, III : la lutte des ouvriers contre l'organisation de l'entreprise capitaliste », dans L'expérience du mouvement ouvrier 2, op. cit., p. 53.

35. Voir ibid., p. 90-91.

36. Voir « Prolétariat et organisation, I », op. cit., p. 74.

37. Voir « Le mouvement révolutionnaire sous le capitalisme moderne », p. 105-108.

38. Voir «Sur le contenu du socialisme, II », op. cit., p. 111 : «Toute société d'exploitation vit parce que ceux qu'elle exploite la font vivre. Mais les esclaves ou les serfs font vivre les maîtres et les seigneurs en conformité avec les normes de la société des maîtres et des seigneurs. Le prolétariat fait vivre le capitalisme à l'encontre des normes du capitalisme. C'est en cela que se trouve l'origine de la crise historique du capitalisme, c'est en cela que le capitalisme est une société grosse d'une perspective révolutionnaire "

39. Texte dactylographié inédit, La mise en question de l'imaginaire dans l'histoire effective, 1963, dans Histoire et création. Textes philosophiques inédits 1945-1967, Paris, Seuil, 2009, p. 200-201.

40. Voir ibid.

41. Voir « Le mouvement révolutionnaire sous le capitalisme moderne », op. cit., p. 106.

42. Voir «Sur le contenu du socialisme, III : la lutte des ouvriers contre l'organisation de l'entreprise capitaliste », op. cit., p. 38.

43. Ibid., p. 53.

44. Voir « L'histoire du mouvement ouvrier », op. cit., p. 90-9. Voir également « Sur le contenu du socialisme, II », op. cit., p. 110-111.

45. «Sur le contenu du socialisme, III : la lutte des ouvriers contre l'organisation de l'entreprise capitaliste », op. cit., p. 71-72. Voir également « Sur le contenu du socialisme, II », op. cit., p. 108.

46. Voir « Sur le contenu du socialisme, II », op. cit., p. 132 ; «Sur le contenu du socialisme, III : la lutte des ouvriers contre l'organisation de l'entreprise capitaliste », op. cit., p. 15-16.

47. Voir « Prolétariat et organisation, I », op. cit., p. 147.

48. «Sur le contenu du socialisme, III : la lutte des ouvriers contre l'organisation de l'entreprise capitaliste », op. cit., p. 74. 


\section{AUTEUR}

NICOLAS POIRIER

Docteur en science politique. Professeur de philosophie, Lycée Montesquieu, Herblay. Nicolas Poirier est notamment l'auteur de Castoriadis, l'imaginaire radical, PUF, 2004. 